\title{
Properties of Nanocelluloses and Their Application as Rheology Modifier in Paper Coating
}

\author{
Chao Liu, ${ }^{\dagger}$ Haishun Du, ${ }^{\dagger}$ Lv Dong, ${ }^{\dagger,}$ Xin Wang, ${ }^{\S}$ Yuedong Zhang, ${ }^{\dagger}$ Guang Yu, ${ }^{\dagger}$ Bin Li, ${ }^{*}, \oplus$ \\ Xindong $\mathrm{Mu},{ }^{\dagger}$ Hui Peng, ${ }^{*},{ }^{\dagger}$ and Huizhou Liu ${ }^{\dagger}$
}

${ }^{\dagger}$ CAS Key Laboratory of Bio-Based Materials, Qingdao Institute of Bioenergy and Bioprocess Technology, Chinese Academy of Sciences, Qingdao, Shandong 266101, China

${ }^{\ddagger}$ University of Chinese Academy of Sciences, Beijing 100049, China

${ }^{\S}$ Department of Marine Chemistry and Geochemistry, College of Ocean and Earth Sciences, Xiamen University, Xiamen 361005, China
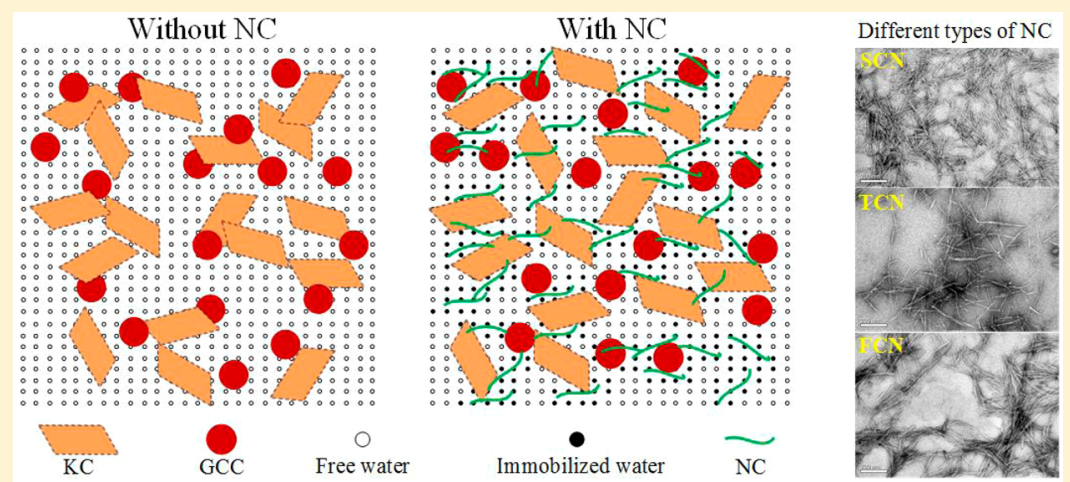

ABSTRACT: In this study, different nanocellulose (NC) products were manufactured from corncob residue (CCR) through sulfuric acid hydrolysis, formic acid hydrolysis, and TEMPO-mediated oxidation methods (the products were referred as SCN, FCN, and TCN, respectively). The properties of NC products and their impact on rheological behavior of paper coatings were comparatively studied. Results showed that compared to SCN and TCN, FCN exhibited large dimensions, limited negative surface charge, and poor stability in their aqueous suspensions, while the FCN aqueous suspension displayed the highest viscoelastic modulus due to the formation of highly entangled network. In paper coatings, SCN exhibited superior thickening and promoted rheological function due to their highly charged surface and strong interactions with pigments and immobilized water molecules, in comparison with other NC products. This study verified that the NC derived from CCR could be utilized as green and renewable additives to improve rheological properties for paper coatings.

\section{INTRODUCTION}

Cellulose is the most abundant biopolymer on the earth and an important source for the manufacture of sustainable materials. ${ }^{1}$ Nanocellulose (NC) is a cellulosic material with nanosize at least in one dimension and can be prepared from natural cellulose by many "top-down methods" (through the process from normal-size cellulosic fibers to nanofibers). Acid hydrolysis (like sulfuric acid hydrolysis), (2,2,6,6-tetramethylpiperidin-1-yl)oxyl (TEMPO)-mediated oxidation, mechanical treatment (e.g., high pressure homogenization), and enzymatic hydrolysis treatment can be adopted to produce $\mathrm{NC}$, but the combined methods (like sulfuric acid hydrolysis plus post mechanical treatment) are usually more cost-effective. ${ }^{2,3} \mathrm{NC}$ has unique properties, such as high aspect ratio, large surface area, light weight, and renewability. Therefore, in recent years, the preparation and application of NC have been paid more and more attention, and $\mathrm{NC}$ has a wide range of potential applications, including reinforcing agent, ${ }^{4}$ sizing agent, ${ }^{5}$ drug delivery, ${ }^{6,7}$ transparent film, ${ }^{8}$ electronic template, ${ }^{9}$ and many others. $^{10}$

NC usually is high aspect ratio nanofiber dispersing in aqueous medium. Due to the hygroscopic character and the strong interconnections of nanofibers, the NC suspensions usually exhibit high viscosity at low solid content (e.g., at 1-2 wt \%), ${ }^{11}$ and they are gel-like suspensions and their thixotropic behavior depends on the morphology and surface charge of fibers. $^{12,13}$ Rheological behavior of NC suspensions has been measured in the past several years. Investigation of the preparation methods, morphology, and rheology of NC products could not only help to understand the microstructure and exploit the possible applications of $\mathrm{NC}$ but also provide

Received: April 29, 2017

Revised: June 24, 2017

Accepted: July 3, 2017

Published: July 3, 2017 
useful information for storage, transportation, and further processing. ${ }^{14}$ The performance of NC products depends on their features, like aspect ratio, surface charge, dispersion, and microstructure, which can be strongly affected by preparation methods and origin of raw materials. ${ }^{15}$ The sulfuric acid hydrolysis and TEMPO-mediated oxidation are the two most well-known methods for NC production. The rheological behavior of these two kinds of NC suspensions has been investigated in the literature. ${ }^{10,16,17}$ Most recently, formic acid (FA) was used to replace mineral acids to manufacture NC (the prepared NC was referred as FCN) because FA could be easily recovered and cause less equipment corrosion. ${ }^{18,19}$ However, there is no investigation dealing with rheological properties of FCN suspensions and no comparative study on the rheology between FCN and the traditional NC products (like the one prepared by sulfuric acid hydrolysis).

NC fabricated from different native cellulose usually shows distinctive rheological properties because of the differences in surface properties, crystallinity, and microstructure. ${ }^{11}$ Corncob residue (CCR), a lignocellulosic waste derived from the furfural and xylose-based products industry, is abundant and lowcosting. In China, about 23 million tons of CCR are generated annually, and burning to recover heat is the traditional way to treat CCR, but burning causes environmental problems and waste of materials with high content of cellulose (>50 wt $\%) .^{20,21}$ Therefore, high-value utilization of CCR is worthy of concern.

Paper coatings are usually mixture of water, inorganic pigments (e.g., kaolin and calcium carbonate), synthetic or natural binders, and other additives, ${ }^{22}$ and paper coatings are mainly used to improve the surface properties of paper and paperboard. The rheological properties of paper coating have great influence on the quality of coated paper, mainly depending on the interactions between binders and pigments. ${ }^{23}$ In general, an addition of thickener or rheology modifier is needed to obtain suitable rheology. ${ }^{24}$ For instance, carboxymethylcellulose (CMC) is commonly used as a thickener and cobinder to alter the rheological properties of coating, which in turn affects the end quality of final coating layer. $^{25}$

NC can bind water strongly on the charged fibrils surface and within the fibrillary aggregates. Its suspension has high viscosity at low solid content owing to its large specific surface area and considerable amount of interactions between NC particles, making them good additives for rheology control. Furthermore, NC suspensions exhibit shear-thinning behavior, which can be caused by breakdown and the change of the viscoelastic structure (i.e., gel-like agglomerates) of the suspensions under shear force. ${ }^{26}$ During paper coating process, the viscosity of paper coating containing NC is relatively low at high shear rates. After transferred to paper surface, the NC in paper coating would help to form a highly viscous layer when shear force ends and affect the dewatering of coating color. ${ }^{26}$ Thus, the thixotropic properties of NC suspension might be an advantage for distributing and thereafter retaining the coating color on paper surface. On the basis of these intrinsic characteristics as well as its biodegradable nature, NC is expected to be a sustainable and promising additive in paper coating formulation. $^{27}$

The objectives in this study included the following:.

(1) Obtain different NC products with different morphologies and charges from CCR by the use of sulfuric acid hydrolysis, TEMPO-mediated oxidation, and formic acid hydrolysis, respectively.

(2) Investigate the impact of morphologies and surface chemical properties of $\mathrm{NC}$ on the rheological properties of $\mathrm{NC}$ suspensions and the paper coatings containing different NC products.

(3) Validate whether the NC derived from CCR could be used as rheology modifier in paper coating.

\section{EXPERIMENTAL SECTION}

2.1. Materials. CCR was a gift from ShengQuan Group, China. The main components of CCR were $63.5 \%$ cellulose, $2.7 \%$ xylan, $25.8 \%$ lignin, and $2.1 \%$ ash based on oven-dried weight, determined according to the National Renewable Energy Laboratory (NREL) procedure. $^{28}$ Sulfuric acid, FA, ferric chloride, sodium hypochlorite, uranyl acetate, sodium bromide, starch, and CMC were purchased from Sinopharm Group Co. Ltd., China. Kaolin clay (KC) and ground calcium carbonates (GCC) were supplied by Ai Keda Chemical Technology Co. Ltd., China. (2,2,6,6-Tetramethylpiperidin-1yl)oxyl (TEMPO) was purchased from Sigma-Aldrich. All chemicals were used as received.

2.2. Preparation of NC. The CCR was treated with an aqueous 3 wt $\% \mathrm{NaOH}$ solution for $3 \mathrm{~h}$ at $100{ }^{\circ} \mathrm{C}$ and then washed until neutrality with tap water. Subsequently the insoluble residue was further bleached with sodium hypochlorite $\left(0.02 \mathrm{~g}\right.$ per gram CCR) at $80^{\circ} \mathrm{C}$ for $2 \mathrm{~h}$ and the $\mathrm{pH}$ value of solution was adjusted to 12 by addition of sodium hydroxide before bleaching. The CCR concentration for bleaching was 5 wt $\%$, and the resulting solid was also washed until neutrality with tap water. The bleached CCR with cellulose and lignin content of $91.6 \%$ and $1.1 \%$, respectively, was air-dried and stored at room temperature for extraction of NC. The NC products were then produced via sulfuric acid hydrolysis, TEMPO-mediated oxidation, and FA hydrolysis, respectively.

2.2.1. Sulfuric Acid Hydrolysis. The bleached CCR was mixed with 64 wt \% sulfuric acid solution preheated to $45^{\circ} \mathrm{C}$ at a liquid-to-solid ratio of $20 \mathrm{~mL} / \mathrm{g}$ under vigorous stirring for 1 h. ${ }^{29}$ Upon completion, the hydrolysis was stopped by 6 -fold cold deionized water. The obtained suspension was washed 4 times followed by centrifugation $(10000 \mathrm{rpm}$ for $5 \mathrm{~min}$ ) and then dialyzed against deionized water until the $\mathrm{pH}$ value remained unchanged. The obtained product was referred as SCN.

2.2.2. TEMPO-Mediated Oxidation. The bleached CCR was suspended in water containing TEMPO $(1.6 \mathrm{mg} / \mathrm{L})$ and sodium bromide $(1 \mathrm{~g} / \mathrm{L})$ with constant stirring at a liquid-tosolid ratio of $100 \mathrm{~mL} / \mathrm{g}$. The $\mathrm{pH}$ of the reaction system was adjusted to 10 via the addition of $0.5 \mathrm{M} \mathrm{NaOH}$. The TEMPOmediated oxidation was started by dropwise adding $45 \mathrm{~mL}$ of aqueous $\mathrm{NaClO}$ solution (10 mmol per gram of cellulose) at ambient condition. The $\mathrm{pH}$ was maintained at 10.5 by adding $0.5 \mathrm{M} \mathrm{NaOH}$ until no additional $\mathrm{NaOH}$ needed, and then it was fully quenched by addition of $800 \mathrm{~mL}$ of anhydrous ethanol. ${ }^{30}$ After that, the obtained cellulosic solid was thoroughly washed with deionized water by centrifugation and passed through a GYB40-10S high-pressure homogenizer (Shanghai Donghua High Pressure Homogenizer Factory, China) 3 times at 300 bar and concentration of 0.2 wt \%. The resultant gel-like solid was referred as TCN.

2.2.3. FA Hydrolysis. The procedure was based on our previous study with slight modifications. ${ }^{17}$ Briefly, the bleached 
CCR and $10 \%$ ferric chloride (based on the dry weight of the bleached CCR) were mixed with $88 \mathrm{wt} \% \mathrm{FA}$ at a liquid-to-solid ratio of $20 \mathrm{~mL} / \mathrm{g}$, and then the mixture was placed in an oil bath at $100{ }^{\circ} \mathrm{C}$ for $6 \mathrm{~h}$ with magnetic stirring at $200 \mathrm{rpm}$. Upon completion, the flask was immediately cooled down by cold running water. Then, the mixture was centrifuged (1000 rpm for $6 \mathrm{~min}$ ), and the separated solid residue was washed by deionized water at least 6 times followed by centrifugation (1000 rpm for $6 \mathrm{~min}$ ) to remove excess acid. At last, the washed solid was passed through a high-pressure homogenizer (GYB40-10S, Shanghai Donghua High Pressure Homogenizer Factory, China) 3 times at $300 \mathrm{bar}$ and concentration of $0.2 \mathrm{wt}$ $\%$. The resultant gel-like solid was referred as FCN.

2.3. Preparation of Coating Colors. KC and GCC were used as pigments to form a standard blend. The model paper coatings were prepared by mixing NC with starch, GCC, and KC. Component amounts were given in parts per 100 (pph, by weight) based on 100 parts by weight of pigments. The relative amount of each ingredient in the coating color formulations is shown in Table S1 in Supporting Information. The coating formation of color mainly consisted of KC 70 pph, GCC 30 $\mathrm{pph}$, and starch $6 \mathrm{pph}$. The coating containing $0.4 \mathrm{pph} \mathrm{CMC}$ as sole additive was referred as control. In other coatings, CMC was totally replaced by different amounts of NC. The amounts of NC in the coating color formulations were $0.05,0.1,0.2$, and $0.4 \mathrm{pph}$, respectively, and the corresponding coatings containing SCN, TCN, and FCN were referred as SCN0.05, SCN0.1, SCN0.2, SCN0.4, TCN0.05, TCN0.1, TCN0.2, TCN0.4, FCN0.05, FCN0.1, FCN0.2, and FCN0.4, respectively. The pigments used in this study were obtained as predispersed pigment slurries. The starch was added to the slurry after cooking in a water bath at $95{ }^{\circ} \mathrm{C}$ for about $20 \mathrm{~min}$, and then all the ingredients were mixed under vigorous agitation. Solids content (including pigments and starch) of the coating colors was $50 \%$ by dry weight. The $\mathrm{pH}$ value of coating color was adjusted to 9.0 using $1 \mathrm{M} \mathrm{NaOH}$ before analysis.

2.4. Morphological Structure Analysis. Transmission electron microscopy (TEM) images were obtained on a field emission H-7600 electron microscope (Japan) at $80 \mathrm{kV}$ accelerating voltage. Dilute NC suspensions with concentration of 0.03 wt \% were deposited on carbon coated TEM grids. After drying, the specimen was stained with 2 wt \% uranyl acetate solution for $10 \mathrm{~min}$. Then the excess staining solution was removed by bibulous paper and the sample was dried at ambient condition before TEM observation. Atomic force microscopy (AFM) analysis was conducted by an Agilent 5400 AFM (Agilent Technologies, Inc., USA), and the measurements were accomplished with a Si cantilever, operating in the tapping mode. Several drops of the suspension (0.01 wt \%) were deposited onto freshly cleaved mica substrates and allowed to dry. Samples were measured at ambient condition. The diameter and length of NC were estimated by analyzing AFM images. A minimum of 200 measurements were made to evaluate the fiber dimension for each type of NC.

2.5. Particle Size Distribution and $\zeta$ Potential. The particle size distributions of NC were measured with dynamic light scattering (DLS), using the photon correlation spectroscopic technique with a Zetasizer Nano S90 (Malvern Instruments Ltd., U.K.). Prior to measurement, the samples were diluted with deionized water to a solid content of $0.01 \mathrm{wt}$ $\%$. The $\zeta$ potentials of NC were detected by a microscopic electrophoresis apparatus (Shanghai Zhongchen Digital Technic Apparatus Co., China) at solid concentration of 0.05 wt \% and were calculated based on the electrophoretic mobility. All the sample suspensions were ultrasonically treated for $10 \mathrm{~min}$ before analyses. For each sample, both the particle size distribution and $\zeta$ potentials were measured 6 times and the average data were reported.

2.6. X-ray Diffraction (XRD) Analysis. XRD was conducted using Bruker D8 ADVANCE X-ray diffractometer (Bruker Co., Germany) equipped with Ni-filtered $\mathrm{Cu} \mathrm{K} \alpha$ radiation generated at $40 \mathrm{kV}$ and $40 \mathrm{~mA}$. The data were collected at an angular range of $4^{\circ} / \mathrm{min}$. The crystallinity index (CrI) was calculated according to the Segal's method: ${ }^{31}$

$$
\mathrm{CrI}=\frac{I_{002}-I_{\text {amorph }}}{I_{002}} \times 100 \%
$$

where $I_{002}$ is the maximum of the diffraction intensity (the 002 plane) and $I_{\text {amorph }}$ is the minimum diffraction intensity (between 101 and 002 peaks).

2.7. Rheological Measurements. Rheological properties of NC suspensions and coating colors were measured using a rheometer (MCR 102, Anton Paar, Austria). The steady-state viscosity of NC suspensions and coating colors were performed in shear rates ranging from 0.01 to $1000 \mathrm{~s}^{-1}$ and from 0.01 to $2000 \mathrm{~s}^{-1}$, respectively. Dynamic viscoelastic moduli, storage modulus $\left(G^{\prime}\right)$ and loss modulus $\left(G^{\prime \prime}\right)$, were measured over frequency in the range of $0.1-10 \mathrm{~Hz}$. To perform the frequency sweep test, the linear viscoelastic range of the sample was obtained from an amplitude sweep at a fixed frequency of $1 \mathrm{~Hz}$. A $50 \mathrm{~mm}$ stainless steel parallel plate was used for the tests and the plate gap was $1 \mathrm{~mm}$. All experiments were carried out at a constant temperature of $23{ }^{\circ} \mathrm{C}$.

\section{RESULTS AND DISCUSSION}

3.1. Morphology and Physicochemical Properties of NC. The bleached CCR was cellulose-rich white powder with size up to more than $100 \mu \mathrm{m}$ as shown in Figure 1a and Figure 1b. The morphologies and dimensions played an important role in the performance of NC. TEM and AFM analyses were used for morphology characterization of NC products (Figure 1ch). AFM was thought to be more widely accessible than TEM. $^{32}$ Therefore, the AFM images were used for evaluating dimensions of NC, and the data are summarized in Table 1. The bleached CCR was disintegrated into NC after acid hydrolysis and TEMPO-mediated oxidation, respectively. As seen from Figure $1 \mathrm{c}-\mathrm{h}$, the dimensions of $\mathrm{NC}$ depended strongly on the processing methods, and the obtained products exhibited distinct characteristics. Sulfuric acid hydrolysis produced well-defined rod-like particles (Figure 1c and Figure 1d), and TEMPO-mediated oxidation yielded thinner and longer nanofiber with much higher aspect ratio (Figure 1e and Figure 1f). Also, the size of NC depended strongly upon the processing intensity. FA hydrolysis produced NC with largest diameter and nearly twice the length of SCN. The larger size of FCN might be due to the relatively weak acidity of FA. ${ }^{33}$

The effects of processing method on the cellulose crystal structure were also investigated, and the XRD curves of the bleached CCR and NC are shown in Figure 2. All the samples exhibited diffraction peaks at approximately $16^{\circ}$ and $22.6^{\circ}$. Acid hydrolysis and TEMPO-mediated oxidation did not cause change in the diffraction peak position, revealing that the crystalline structure of cellulose was not altered. ${ }^{34}$ However, the CrI was changed after the treatments. The CrI values of SCN and TCN were lower than that of the bleached CCR. 


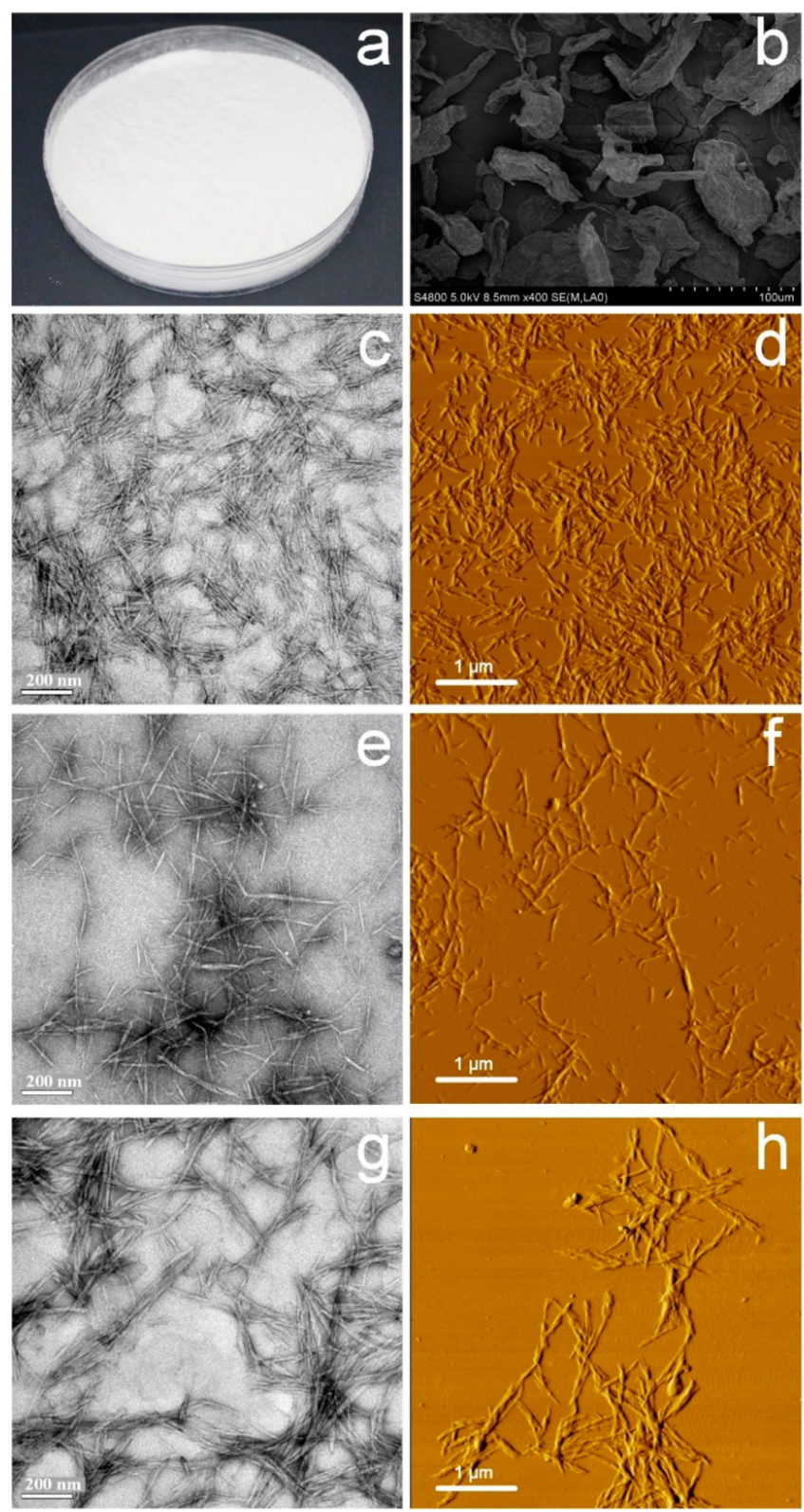

Figure 1. Morphologies of bleached CCR and NC: photographs of bleached CCR (a) and SEM image of bleached CCR (b); TEM images of SCN, TCN, and FCN (c, e, g); AFM images of SCN, TCN, and FCN $(d, f, h)$. The scale bars of TEM and AFM images were $200 \mathrm{~nm}$ and $1 \mu \mathrm{m}$, respectively.

Generally, strong acid hydrolysis could remove the amorphous areas and resulted in a higher crystallinity, ${ }^{35}$ but part of the original cellulose structure of CCR might be dissociated during the industrial hemicellulose extraction process, leading to the fact that the damaged crystalline regions of cellulose were more

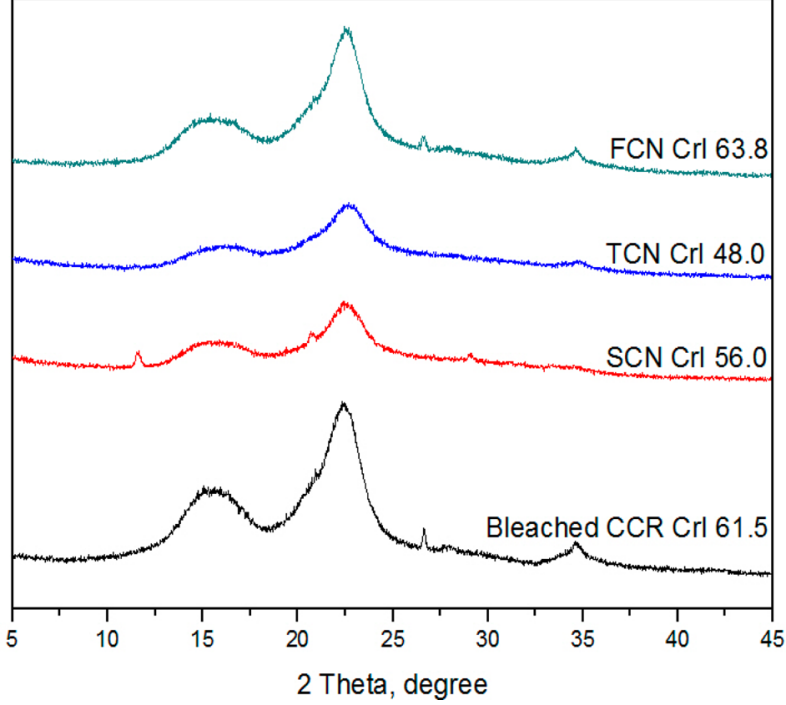

Figure 2. XRD patterns and CrI of the bleached CCR and NC products.

accessible to the acid hydrolysis. ${ }^{19}$ Similarly, the oxidation could also cause damage to the ordered crystalline structure of the bleached CCR during NC production. ${ }^{36} \mathrm{FA}$ is the simplest carboxylic acid with relatively weak acidity. The corresponding CrI changing from $61.5 \%$ (the bleached CCR) to $63.8 \%$ (FCN) indicated that FA hydrolysis exhibited better selectivity and more crystalline zone was reserved. ${ }^{15,37}$

It is well-known that SCN is dispersible in water due to the existence of surface sulfate ester groups introduced during hydrolysis. ${ }^{32}$ Similarly, TEMPO-mediated oxidation modified the cellulose surface with oxidation of primary hydroxyls to carboxylate groups. ${ }^{38}$ The surface charge of NC products resulting from the presence of sulfate and carboxylate groups could be measured roughly by $\zeta$ potential which reflected the stability of colloidal suspensions. Due to the relatively high absolute values of $\zeta$ potential, the suspensions of SCN and TCN exhibited good stability for $24 \mathrm{~h}$ (Figure 3 ). However, during FA treatment, hydrolysis and esterification did not generate adequate charged chemical groups on the NC surface. Thus, flocculation of FCN could be observed within $24 \mathrm{~h}$ due to its low absolute value of $\zeta$ potential $(-10.5 \pm 2.1 \mathrm{mV})$.

Birefringence analysis is usually used to confirm the presence of isolated cellulose nanofibers in aqueous suspension, and it is considered to be an effective way to prove good dispersibility of NC suspensions. ${ }^{34}$ The flow birefringence properties of NC suspension at $0.3 \mathrm{wt} \%$ concentration were observed between the crossed polarizers in shearing conditions (Figure 3 ). The presence of birefringence phenomenon indicated that the NC particles formed a chiral nematic liquid crystalline phase in equilibrium with isotropic phase, indicating a good dispersion for SCN and TCN products. In most cases, birefringence is

Table 1. Dimensions, $\zeta$ Potentials, and Average Particles Size of NC

$\begin{array}{ccccc}\text { product } & \text { length, } \mathrm{nm}^{a} & \text { diameter, } \mathrm{nm}^{a} & \text { aspect ratio } & \zeta \text { potential, mV }{ }^{a} \\ \text { SCN } & 206 \pm 65 & 5.4 \pm 1.8 & 38 \pm 17 & -33.8 \pm 1.7 \\ \text { TCN } & 382 \pm 118 & 3.8 \pm 0.9 & 101 \pm 85 & -23.1 \pm 2.3 \\ \text { FCN } & 421 \pm 112 & 6.5 \pm 2.0 & 67 \pm 32 & -10.5 \pm 2.1\end{array}$

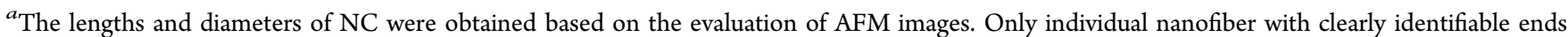
was measured, and at least 200 measurements were conducted for each type of sample. ${ }^{b}$ Average particle sizes were calculated based on PSD data. 

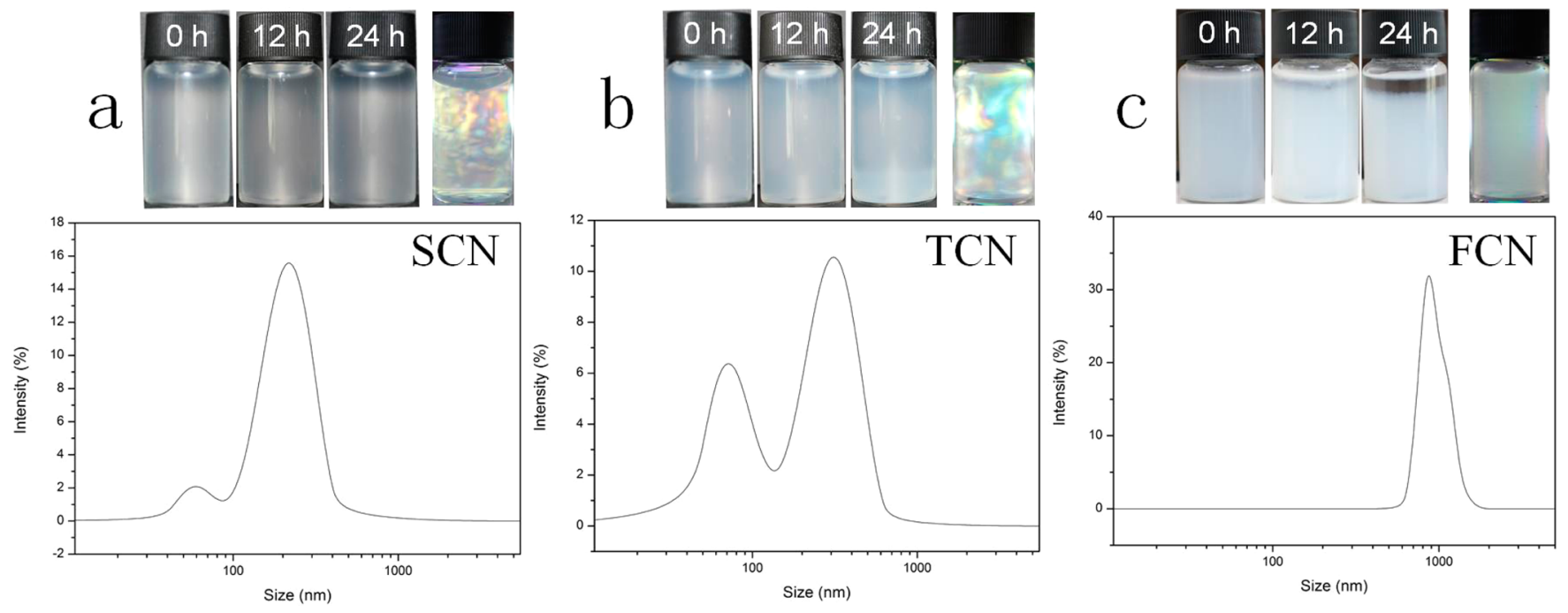

Figure 3. Dispersion state, shear-induced birefringence (upper) and particle size distributions (lower) of SCN (a), TCN (b), and FCN (c) in aqueous solution.
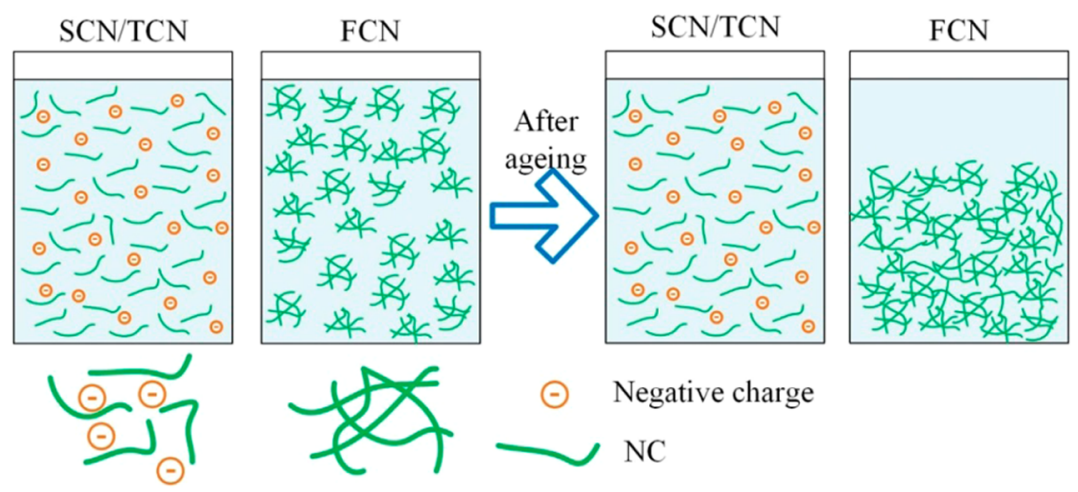

Figure 4. Schematic plot of the dispersion state for different NC products in aqueous solution.

happening only with cellulose nanocrystals, but TCN suspension also exhibited clear birefringence, probably owing to the highly anionic groups and the post mechanical disintegration. $^{39}$ This phenomenon indicated an individual nanofiber dispersion. As reported previously, birefringence was also observed with the CNF prepared by TEMPO-mediated oxidation method from wood source. ${ }^{39-41}$ However, FCN suspension did not show the phenomenon because of the flocculation of FCN particles. On the other hand, DLS method was conducted to give a quick analysis of the rough particle size distribution (PSD) (i.e., the average of agglomerate sizes of NC particles). As shown in Figure 3, the TCN had a wider PSD in comparison with SCN, but the average particle sizes of SCN and TCN were similar to the nanofiber lengths measured based on AFM analyses. For FCN sample, its average particle size was much larger than its length measured with AFM. This result also verified the flocculation of FCN particles, as schematically displayed in Figure 4. These distinctive features, including morphology and surface properties of NC samples, might lead to different performance when they are used as rheology modifier.

3.2. Rheological Behavior of NC Suspensions. The rheological behaviors of NC suspension are strongly dependent on their characteristics, such as dimensional size, particles size, surface charge, and dispersion microstructure. Hence, before use of NC as rheology modifier for paper coating, the rheology of NC suspensions was determined. Figure 5a shows apparent viscosity as a function of shear rate for NC aqueous suspensions at the same concentration of $4 \mathrm{wt} \%$. All suspensions exhibited "shear-thinning" behavior, as already known from early works on NC suspensions. ${ }^{42}$ This phenomenon was due to the fact that the network constructed by strong hydrogen or ionic bonding interactions among NC particles was broken by increased shear strength. ${ }^{43}$ In comparison with TCN suspension, SCN suspension presented more significant shear-thinning behavior (the dashed lines represent fitting straight line in Figure 5). SCN suspension displayed higher viscosity at the low shear rates but lower viscosity at the high shear rates compared to TCN suspension. The highly charged surface could make SCN bind more water molecules and then increased the viscosity. But the formed network by SCN and water molecules was fragile to shear force, due to the short length and low aspect ratio of SCN, while TCN with larger fiber length and aspect ratio could form more stable network thus exhibiting higher viscosity at the high shear rates. Figure 5a also shows that FCN suspension displayed much higher viscosity at low shear rate than SCN and TCN. For example, at a shear rate of $0.1 \mathrm{~s}^{-1}$, the viscosities of SCN, TCN, and FCN were 40.6, 18.7, and $85.4 \mathrm{~Pa} \cdot \mathrm{s}$, respectively. This was because FCN particles with lower surface charge and much longer fiber length were easily aggregated and entangled to form a relatively strong network. However, a sudden increase of viscosity for 

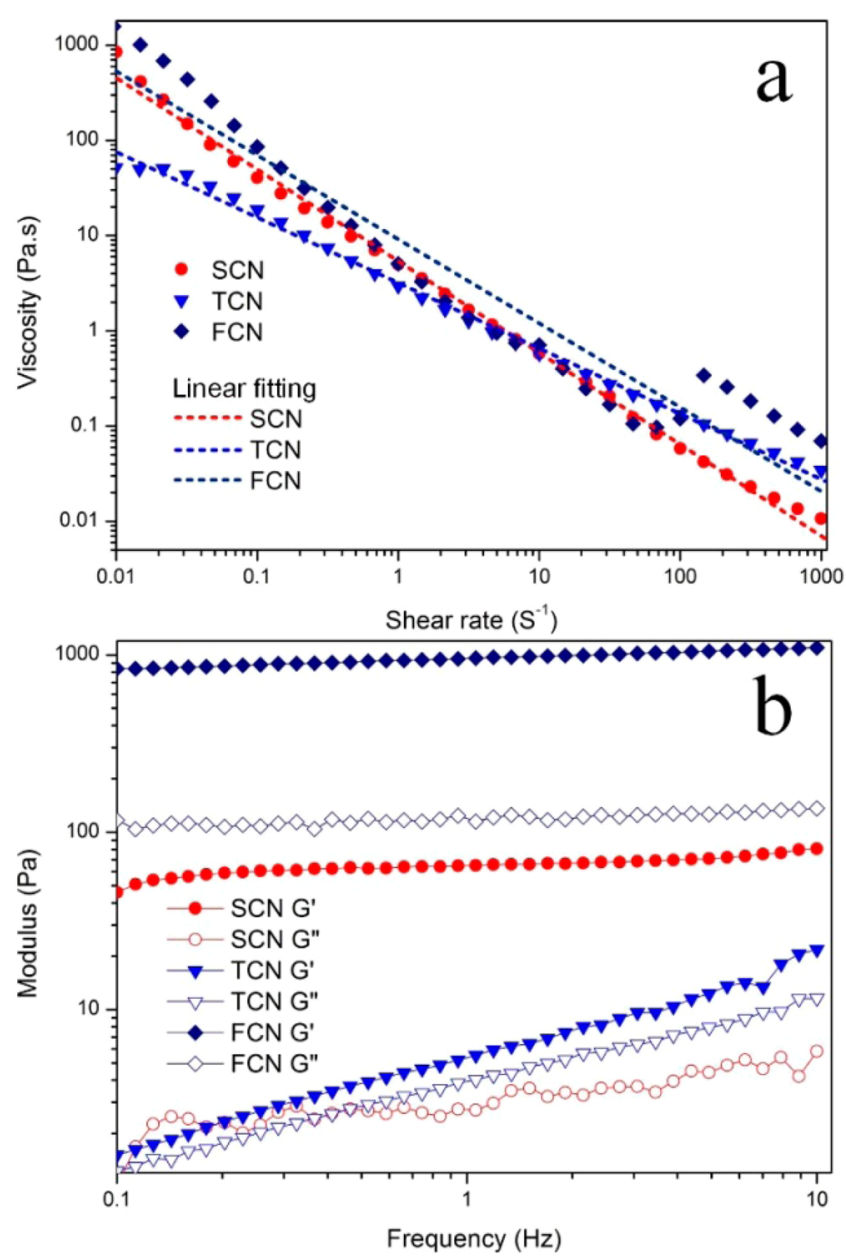

Figure 5. Rheological behaviors of NC suspensions: viscosity versus shear rate (a); modulus versus frequency (b). $G^{\prime}$ is dynamic storage modulus. $G^{\prime \prime}$ is dynamic loss modulus.

FCN suspension occurred at the shear rate of about $100 \mathrm{~s}^{-1}$, which was because FCN particles were rearranged to form an ordered network again. Similar results were also reported in literature for the SCN suspensions with the concentration of $0.91-3.17$ wt $\%{ }^{43}$

Figure $5 \mathrm{~b}$ illustrates the $G^{\prime}$ and $G^{\prime \prime}$ of NC suspensions as a function of frequency at a fixed strain of $0.05 \%$. Both $G^{\prime}$ and $G^{\prime \prime}$ displayed a gradual increase toward higher frequency. All $G^{\prime}$ values were much larger than $G^{\prime \prime}$ indicating a solid-like elastic behavior of the typical material for a gel-like structure. The similar rheological behaviors were observed by other researchers. ${ }^{14}$ The sulfuric acid hydrolysis resulted in shorter nanofiber compared to the TEMPO-mediated oxidation and FA hydrolysis, and the reduction of nanofiber length could reduce the possibility of entanglements, but more surrounding water molecules could be immobilized onto the surface of SCN. Thus, the reduction of the gel stiffness caused by the reduction of the nanofiber length could be compensated, which enabled maintaining of the formation of a relatively stable network and resulted in a higher $G^{\prime}$ for SCN suspension (compared to TCN). ${ }^{44}$ Figure $5 \mathrm{~b}$ also shows that FCN suspensions had much higher $G^{\prime}$ compared to SCN and TCN suspensions, indicating that FCN suspensions had a more pronounced gel-like structure. FCN had low $\zeta$ potential (absolute value), much larger length, and higher flexibility. Thus, although the electrostatic repulsions among FCN particles and the hydrogen bonds between FCN and water molecules were very weak, the physical entanglements between FCN particles were relatively strong. That is to say, the physical entanglements between FCN particles were the primary contributor to the stiffening of network in the suspensions. In the present study, sulfuric acid hydrolysis and TEMPO-mediated oxidation cut down the length of nanofibers, introduced negative charges, and produced more individualized nanofibers. The network structure of SCN and TCN suspension was dominated by electrostatic force and immobilized water molecules rather than the physical entanglements. Therefore, due to the higher degree of entanglements, FCN particles could form relatively strong network which was more resistant to mechanical shearing forces. $^{15,44}$

The rheological properties of coating colors are very important for controlling transfer of pigments to paper surface. At high shear rates the viscosity of the NC suspension can be kept relatively low. When the coating containing $\mathrm{NC}$ is transferred to the paper and the shear forces end, a highly viscous layer could be formed. Furthermore, the rheological properties also have significant influences on the blade application and the dewatering behavior of paper coating. Therefore, NC products could be used in paper coating formulations due to their biodegradable nature, viscoelasticity, and strong shear-thinning properties. ${ }^{45}$

3.3. Rheology of Paper Coating Containing NC. Paper coating colors are commonly shear-thinning non-Newtonian fluids (viscosity decreases with the increase of shear rate). As shown in Figure 6, all coating colors showed a similar pattern of shear-thinning behavior and exhibited the highest viscosity at low shear rate. The addition of NC significantly increased the viscosity compared to the control sample at low shear rates and led to more obvious shear-thinning behavior. Moreover, the coatings containing FCN displayed less pronounced shearthinning behavior than the ones containing SCN and TCN. The coatings containing SCN exhibited highest viscosity and the ones containing FCN exhibited lowest viscosity at the same $\mathrm{NC}$ loading in the low shear rate region. Also, at a given shear rate, the viscosity of the paper coatings containing $\mathrm{NC}$ was clearly higher compared to the control (with 0.4 wt \% CMC) (Table S2). This indicated that NC could much more effectively increase the viscosity of paper coatings at low shear rates compared to CMC. With the increase of shear rate, the viscosity changes of all coatings tended to be consistent and the addition of NC could not result in a clear increase of viscosity at higher shear rates $\left(>500 \mathrm{~s}^{-1}\right)$. The mechanical shear force at high shear rate can break down the entangled network among the NC particles and pigments, thus reducing the thickening effect of NC.

To identify the response of substitution of CMC with NC to viscoelastic properties of coating colors, oscillatory shear measurements were performed. In Figure 7, both $G^{\prime}$ and $G^{\prime \prime}$ were presented as the functions of frequency at a fixed strain of $0.4 \%$ within the linear viscoelastic region. As observed in the coatings containing NC, $G^{\prime}$ increased with the frequency and it was much larger than $G^{\prime \prime}$ for all coating colors, which showed a viscoelastic solid-like feature, indicating that the elastic properties were dominant compared to the viscous properties. The high frequency could increase the bonding between NC and pigments and the rate of formation of the inherent network, which led to an increase of $G^{\prime}$. The $G^{\prime}$ showed frequency sensitive characteristic at low angular frequency and became more frequency-independent at high frequency. For the 

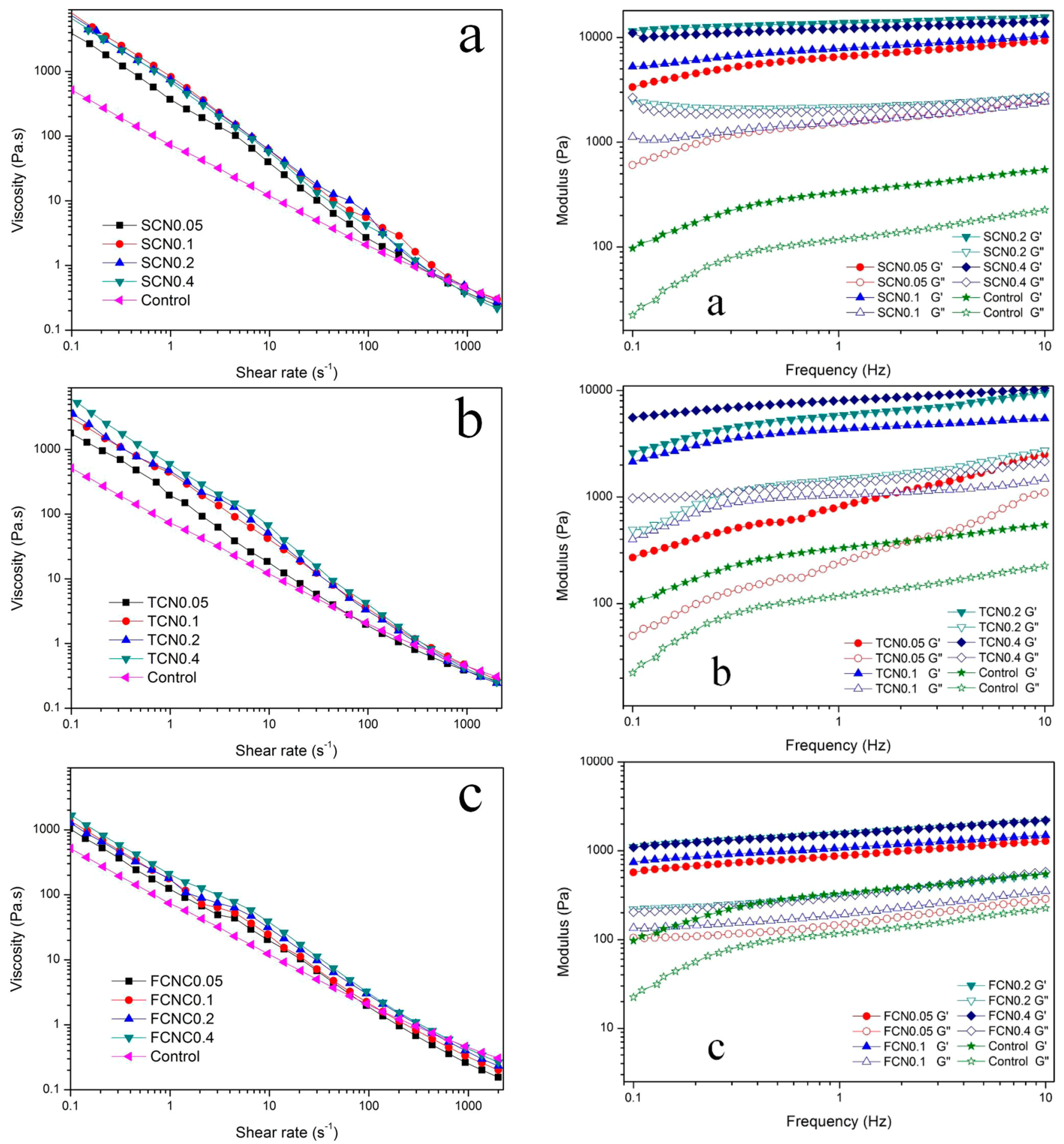

Figure 6. Viscosity versus shear rate for different coating colors: coatings containing SCN (a); coatings containing TCN (b); coatings containing FCN (c).

colors with NC loadings above $0.2 \mathrm{pph}$, the $G^{\prime}$ remained relatively independent of frequency. This was because more nanofibers in the color matrix were helpful to form stronger network structure, which was stable enough to resist the angular frequency. A similar tendency for $G^{\prime \prime}$ values can also be observed in Figure 7. Moreover, the coating containing SCN exhibited lower $G^{\prime \prime}$ value at low frequencies due to its more fluid-like property, while the $G^{\prime \prime}$ increased at the higher frequencies caused by the enhancing interaction among the

Figure 7. $G^{\prime}$ and $G^{\prime \prime}$ of the coatings containing NC (SCN (a), TCN (b), FCN (c)) as a function of frequency.

coating pigments. The similar phenomena were reported in previous studies. $^{26}$

For the coating colors containing TCN and FCN, the $G^{\prime}$ values exhibited a positive correlation with the NC loading, indicating a formation of increasingly stiff network in coatings. This phenomenon was due to the increased interactions between NC and pigments when the NC loading was increased. The coating colors containing SCN exhibited the highest $G^{\prime}$ and $G^{\prime \prime}$. Unlike TCN and FCN, the increasing of SCN loading did not always result in higher $G^{\prime}$ and $G^{\prime \prime}$. For example, the $G^{\prime}$ 
and $G^{\prime \prime}$ values of SCN0.2 were larger than the ones of SCN0.4. The large number of hydroxyl and negatively charged sulfate groups of SCN enabled SCN particles to greatly interact with pigments as well as the adjacent water molecules. But the introduction of sulfate groups on the surface of SCN generated strong electrostatic repulsion, which reduces the interparticle interactions and stability of network structure built by SCN and pigments at high SCN loading. The short length and high mobility, which led to the less possibility of formation of a physically entangled network, also had a negative effect on the stability of network structure. ${ }^{46,47}$

The $G^{\prime}$ and $G^{\prime \prime}$ values of the coatings containing SCN were larger than the ones containing TCN and FCN, mainly due to the formation of intense surface interactions between SCN and coating pigments. SCN had strongly hydrophilic and negatively charged surface generated by hydroxyl and sulfate groups. Thus, SCN could facilitate the dispersion of pigment particles and interaction with inorganic pigments via hydrogen bonds and ionic bonds, particular for the isometric GCC with smaller size compared to the plate-like $\mathrm{KC} .{ }^{48}$ On the other hand, SCN could bind abundant water molecules due to its high surface area and large number of hydroxyl groups and tends to form gel-like structure. The pigments, SCN, and immobilized water molecules bound together to form a tough network, thereby leading to a significant enhancement of $G^{\prime}$ and $G^{\prime \prime}$ values. Conversely, the coating colors containing FCN exhibited the lowest viscosity and modulus. This was because the FCN had less hydroxyl and negatively charged groups (lower absolute $\zeta$ potential, Table 1), which implied weak interactions among FCN, immobilized water molecules, and pigments. However, the lower water binding property of $\mathrm{FCN}$ might benefit to the coating color application in comparison with the SCN with stronger gel nature. ${ }^{49}$ Therefore, the surface chemical properties of NC products played a more important role in rheological modification of paper coating compared to the impact of morphology of NC particles. Previous study also reported that the surface functional groups and interfacial properties were crucial for the application of nanomaterial in paper coating. ${ }^{50}$

The excellent rheological properties are of great importance for the coating performance. Under shearing force, low viscosity of color was highly advantageous to the coating operation. In addition, the high viscosity of coating could maintain stability of pigments dispersion, which is helpful to store. $^{47,50}$

\section{CONCLUSION}

In the present study, sulfuric acid hydrolysis, TEMPO-mediated oxidation, and FA hydrolysis were used to fabricate NC from the bleached CCR, respectively. The obtained FCN exhibited distinctive features with SCN and TCN such as larger dimension, higher crystallinity, and less negative charges on the surface. All NC suspensions showed pronounced shearthinning behavior, but the FCN suspension showed the highest viscosity in low shear rate region and viscoelastic modulus due to its strong physical entanglements forming interconnected networks. On the other hand, the rheological properties of coating colors could be modified significantly by adding a small amount of NC. Compared with the coating containing TCN and FCN, the ones containing SCN exhibited more rigid solidlike viscoelastic properties owing to strong surface interaction among SCN, pigments, and immobilized water molecules. However, the FCN with less charged surface displayed a relatively weak effect on the rheological behavior of paper coating. Therefore, the surface chemical properties of NC played a much more important role compared to the dimensions of NC particles in rheological modification of paper coating, although both morphologies and surface chemical properties of NC have big impact on the rheological behavior of $\mathrm{NC}$ aqueous suspensions. In addition, corncob residue can be used as a source of NC production and such obtained NC can be used as a rheology modifier and partial substitution of binder. The results of this study can provide guidance and useful information for the application of novel NC-based materials, such as well-drilling fluid, paints, and foods.

\section{ASSOCIATED CONTENT}

\section{Supporting Information}

The Supporting Information is available free of charge on the ACS Publications website at DOI: 10.1021/acs.iecr.7b01804.

Coating color formulations and viscosities of coating colors (PDF)

\section{AUTHOR INFORMATION}

\section{Corresponding Authors}

*B.L.: tel, +96-532-80662725; fax, +86-532-80662724; e-mail, libin@qibebt.ac.cn.

*H.P.: e-mail, penghui@qibebt.ac.cn.

ORCID

Bin Li: 0000-0002-8903-3874

\section{Author Contributions}

The manuscript was written through contributions of all authors. All authors have given approval to the final version of the manuscript.

\section{Notes}

The authors declare no competing financial interest.

\section{ACKNOWLEDGMENTS}

The financial support of this work was from the National Natural Science Foundation of China (Grants 31470609, 25106240, and 21433001), Primary Research and Development Plan of Shandong Province (Grants 2016GGX104003 and 2016CYJS07A02), The Young Taishan Scholars Program of Shandong Province (Grant tsqn20161052), The Taishan Scholars Climbing Program of Shandong (Grant tspd20150210), and the National Science \& Technology Pillar Program during the Twelfth Five-Year Plan Period (Grant 2015BAD14B06).

\section{ABBREVIATIONS}

$\mathrm{NC}=$ nanocellulose

TEMPO $=(2,2,6,6$-tetramethylpiperidin-1-yl)oxyl

$\mathrm{FA}=$ formic acid

$\mathrm{CCR}=$ corncob residue

$\mathrm{CMC}=$ carboxymethylcellulose

NREL = National Renewable Energy Laboratory

$\mathrm{KC}=$ kaolin clay

$\mathrm{GCC}=$ ground calcium carbonate

$\mathrm{SCN}=\mathrm{NC}$ prepared by sulfuric acid hydrolysis

$\mathrm{TCN}=\mathrm{NC}$ prepared by TEMPO-mediated oxidation

FCN $=$ NC prepared by formic acid hydrolysis

TEM $=$ transmission electron microscopy

AFM $=$ atomic force microscopy

$\mathrm{XRD}=\mathrm{X}$-ray diffraction 
CrI = crystallinity index

DLS = dynamic light scattering

$G^{\prime}=$ dynamic storage modulus

$G^{\prime \prime}=$ dynamic loss modulus

PSD $=$ particle size distribution

\section{REFERENCES}

(1) Wang, S.; Lu, A.; Zhang, L. Recent advances in regenerated cellulose materials. Prog. Polym. Sci. 2016, 53, 169-206.

(2) Xie, J.; Li, J. Smart drug delivery system based on nanocelluloses. J. Bioresour. Bioprod. 2017, 2 (1), 1-3.

(3) Du, H.; Liu, C.; Wang, D.; Zhang, Y.; Yu, G.; Si, C.; Li, B.; Mu, $\mathrm{X}$.; Peng, H. Sustainable preparation and characterization of thermally stable and functional cellulose nanocrystals and nanofibrils via formic acid hydrolysis. J. Bioresour. Bioprod. 2017, 2 (1), 10-15.

(4) Ng, H.-M.; Sin, L. T.; Tee, T.-T.; Bee, S.-T.; Hui, D.; Low, C.-Y.; Rahmat, A. R. Extraction of cellulose nanocrystals from plant sources for application as reinforcing agent in polymers. Composites, Part B 2015, 75, 176-200.

(5) Yang, L. M.; Lu, S.; Li, J. J.; Zhang, F. S.; Cha, R. T. Nanocrystalline cellulose-dispersed AKD emulsion for enhancing the mechanical and multiple barrier properties of surface-sized paper. Carbohydr. Polym. 2016, 136, 1035-1040.

(6) Zhang, Y. P.; Zhao, Q.; Wang, H. S.; Jiang, X. Y.; Cha, R. T. Preparation of green and gelatin-free nanocrystalline cellulose capsules. Carbohydr. Polym. 2017, 164, 358-363.

(7) Zhao, J.; Lu, C.; He, X.; Zhang, X.; Zhang, W.; Zhang, X. Polyethylenimine-grafted cellulose nanofibril aerogels as versatile vehicles for drug delivery. ACS Appl. Mater. Interfaces 2015, 7 (4), 2607-2615.

(8) Sun, X.; Wu, Q.; Ren, S.; Lei, T. Comparison of highly transparent all-cellulose nanopaper prepared using sulfuric acid and TEMPO-mediated oxidation methods. Cellulose 2015, 22 (2), 11231133.

(9) Tang, Y.; He, Z.; Mosseler, J. A.; Ni, Y. Production of highly electro-conductive cellulosic paper via surface coating of carbon nanotube/graphene oxide nanocomposites using nanocrystalline cellulose as a binder. Cellulose 2014, 21 (6), 4569-4581.

(10) Dimic-Misic, K.; Salo, T.; Paltakari, J.; Gane, P. Comparing the rheological properties of novel nanofibrillar cellulose-formulated pigment coating colours with those using traditional thickener. Nord. Pulp Pap. Res. J. 2014, 29 (2), 253-270.

(11) Nechyporchuk, O.; Belgacem, M. N.; Pignon, F. Current progress in rheology of cellulose nanofibril suspensions. Biomacromolecules 2016, 17 (7), 2311-2320.

(12) Mohtaschemi, M.; Dimic-Misic, K.; Puisto, A.; Korhonen, M.; Maloney, T.; Paltakari, J.; Alava, M. J. Rheological characterization of fibrillated cellulose suspensions via bucket vane viscometer. Cellulose 2014, 21 (3), 1305-1312.

(13) Dimic-Misic, K.; Gane, P. A. C.; Paltakari, J. Micro-and nanofibrillated cellulose as a rheology modifier additive in CMCcontaining pigment-coating formulations. Ind. Eng. Chem. Res. 2013, 52 (45), 16066-16083.

(14) Qiao, C.; Chen, G.; Zhang, J.; Yao, J. Structure and rheological properties of cellulose nanocrystals suspension. Food Hydrocolloids 2016, 55, 19-25.

(15) Li, M.-C.; Wu, Q.; Song, K.; Lee, S.; Qing, Y.; Wu, Y. Cellulose nanoparticles: structure-morphology-rheology relationships. ACS Sustainable Chem. Eng. 2015, 3 (5), 821-832.

(16) Salo, T.; Dimic-Misic, K.; Gane, P.; Paltakari, J. Application of pigmented coating colours containing MFC/NFC: Coating properties and link to rheology. Nord. Pulp Pap. Res. J. 2015, 30 (1), 165-178.

(17) Dimic-Misic, K.; Puisto, A.; Gane, P.; Nieminen, K.; Alava, M.; Paltakari, J.; Maloney, T. The role of MFC/NFC swelling in the rheological behavior and dewatering of high consistency furnishes. Cellulose 2013, 20 (6), 2847-2861.

(18) Du, H.; Liu, C.; Mu, X.; Gong, W.; Lv, D.; Hong, Y.; Si, C.; Li, B. Preparation and characterization of thermally stable cellulose nanocrystals via a sustainable approach of $\mathrm{FeCl}_{3}$-catalyzed formic acid hydrolysis. Cellulose 2016, 23 (4), 2389-2407.

(19) Liu, C.; Li, B.; Du, H.; Lv, D.; Zhang, Y.; Yu, G.; Mu, X.; Peng, $\mathrm{H}$. Properties of nanocellulose isolated from corncob residue using sulfuric acid, formic acid, oxidative and mechanical methods. Carbohydr. Polym. 2016, 151, 716-724.

(20) Xing, Y.; Ji, L.; Liu, Z.; Zhang, W.; Jiang, J. Effects of Gleditsia saponin on high-solids enzymatic hydrolysis of furfural residues. Ind. Crops Prod. 2015, 64, 209-214.

(21) Bu, L.; Xing, Y.; Yu, H.; Gao, Y.; Jiang, J. Comparative study of sulfite pretreatments for robust enzymatic saccharification of corn cob residue. Biotechnol. Biofuels 2012, 5, 87.

(22) Morsy, F. A.; El-Sherbiny, S.; Hassan, M. S.; Mohammed, H. F. Modification and evaluation of Egyptian kaolinite as pigment for paper coating. Powder Technol. 2014, 264, 430-438.

(23) El-Sadi, H.; Carreau, P.; Esmail, N. Changes in the rheological and colloidal properties of paper coating liquids with paper-yellowing inhibition additives. J. Colloid Interface Sci. 2004, 271 (2), 496-503.

(24) Moan, M.; Page, A.; Carreau, P. J.; Heuzey, M. C. Rheological behavior of coating colors: Influence of thickener. Can. J. Chem. Eng. 2002, 80 (6), 1181-1188.

(25) Liang, H.; Long, Z.; Yang, S.; Dai, L. Organic modification of bentonite and its effect on rheological properties of paper coating. Appl. Clay Sci. 2015, 104, 106-109.

(26) Karppinen, A.; Saarinen, T.; Salmela, J.; Laukkanen, A.; Nuopponen, M.; Seppala, J. Flocculation of microfibrillated cellulose in shear flow. Cellulose 2012, 19 (6), 1807-1819.

(27) Dimic-Misic, K.; Gane, P. A. C.; Paltakari, J. Micro- and nanofibrillated cellulose as a rheology modifier additive in CMCcontaining pigment-coating formulations. Ind. Eng. Chem. Res. 2013, 52 (45), 16066-16083.

(28) Sluiter, A.; Hames, B.; Ruiz, R.; Scarlata, C.; Sluiter, J.; Templeton, D.; Crocker, D. Determination of structural carbohydrates and lignin in biomass (LAP); NREL/TP-510-42618; National Renewable Energy Laboratory: Golden, CO, U.S., 2011.

(29) Jiang, F.; Hsieh, Y. L. Cellulose nanocrystal isolation from tomato peels and assembled nanofibers. Carbohydr. Polym. 2015, 122, $60-68$.

(30) Liu, J.; Korpinen, R.; Mikkonen, K. S.; Willför, S.; Xu, C. Nanofibrillated cellulose originated from birch sawdust after sequential extractions: a promising polymeric material from waste to films. Cellulose 2014, 21 (4), 2587-2598.

(31) Segal, L.; Creely, J.; Martin, A.; Conrad, C. An empirical method for estimating the degree of crystallinity of native cellulose using the $\mathrm{X}$ ray diffractometer. Text. Res. J. 1959, 29 (10), 786-794.

(32) Sacui, I. A.; Nieuwendaal, R. C.; Burnett, D. J.; Stranick, S. J.; Jorfi, M.; Weder, C.; Foster, E. J.; Olsson, R. T.; Gilman, J. W. Comparison of the properties of cellulose nanocrystals and cellulose nanofibrils isolated from bacteria, tunicate, and wood processed using acid, enzymatic, mechanical, and oxidative methods. ACS Appl. Mater. Interfaces 2014, 6 (9), 6127-6138.

(33) Martínez-Sanz, M.; Lopez-Rubio, A.; Lagaron, J. M. Optimization of the nanofabrication by acid hydrolysis of bacterial cellulose nanowhiskers. Carbohydr. Polym. 2011, 85 (1), 228-236.

(34) Deepa, B.; Abraham, E.; Cordeiro, N.; Mozetic, M.; Mathew, A. P.; Oksman, K.; Faria, M.; Thomas, S.; Pothan, L. A. Utilization of various lignocellulosic biomass for the production of nanocellulose: a comparative study. Cellulose 2015, 22 (2), 1075-1090.

(35) Chen, W.; Li, Q.; Wang, Y.; Yi, X.; Zeng, J.; Yu, H.; Liu, Y.; Li, J. Comparative study of aerogels obtained from differently prepared nanocellulose fibers. ChemSusChem 2014, 7 (1), 154-161.

(36) Li, B.; Xu, W.; Kronlund, D.; Määttänen, A.; Liu, J.; Smått, J.-H.; Peltonen, J.; Willför, S.; Mu, X.; Xu, C. Cellulose nanocrystals prepared via formic acid hydrolysis followed by TEMPO-mediated oxidation. Carbohydr. Polym. 2015, 133, 605-612.

(37) Du, H.; Liu, C.; Zhang, Y.; Yu, G.; Si, C.; Li, B. Preparation and characterization of functional cellulose nanofibrils via formic acid hydrolysis pretreatment and the followed high-pressure homogenization. Ind. Crops Prod. 2016, 94, 736-745. 
(38) Takaichi, S.; Saito, T.; Tanaka, R.; Isogai, A. Improvement of nanodispersibility of oven-dried TEMPO-oxidized celluloses in water. Cellulose 2014, 21 (6), 4093-4103.

(39) Isogai, A.; Saito, T.; Fukuzumi, H. TEMPO-oxidized cellulose nanofibers. Nanoscale 2011, 3 (1), 71-85.

(40) Shimizu, M.; Fukuzumi, H.; Saito, T.; Isogai, A. Preparation and characterization of TEMPO-oxidized cellulose nanofibrils with ammonium carboxylate groups. Int. J. Biol. Macromol. 2013, 59, 99104.

(41) Tanaka, R.; Saito, T.; Isogai, A. Cellulose nanofibrils prepared from softwood cellulose by $\mathrm{TEMPO} / \mathrm{NaClO} / \mathrm{NaClO}_{2}$ systems in water at $\mathrm{pH} 4.8$ or 6.8. Int. J. Biol. Macromol. 2012, 51, 228-234.

(42) Grüneberger, F.; Künniger, T.; Zimmermann, T.; Arnold, M. Rheology of nanofibrillated cellulose/acrylate systems for coating applications. Cellulose 2014, 21 (3), 1313-1326.

(43) Liu, D.; Chen, X.; Yue, Y.; Chen, M.; Wu, Q. Structure and rheology of nanocrystalline cellulose. Carbohydr. Polym. 2011, 84 (1), 316-322.

(44) Benhamou, K.; Dufresne, A.; Magnin, A.; Mortha, G.; Kaddami, $\mathrm{H}$. Control of size and viscoelastic properties of nanofibrillated cellulose from palm tree by varying the TEMPO-mediated oxidation time. Carbohydr. Polym. 2014, 99, 74-83.

(45) Paakko, M.; Ankerfors, M.; Kosonen, H.; Nykanen, A.; Ahola, S.; Osterberg, M.; Ruokolainen, J.; Laine, J.; Larsson, P.; Ikkala, O.; Lindström, T. Enzymatic hydrolysis combined with mechanical shearing and high-pressure homogenization for nanoscale cellulose fibrils and strong gels. Biomacromolecules 2007, 8 (6), 1934-1941.

(46) Tang, Y.; Hu, X.; Zhang, X.; Guo, D.; Zhang, J.; Kong, F. Chitosan/titanium dioxide nanocomposite coatings: rheological behavior and surface application to cellulosic paper. Carbohydr. Polym. 2016, 151, 752-759.

(47) Lasseuguette, E.; Roux, D.; Nishiyama, Y. Rheological properties of microfibrillar suspension of TEMPO-oxidized pulp. Cellulose 2008, 15 (3), 425-433.

(48) Li, M. C.; Wu, Q.; Song, K.; Qing, Y.; Wu, Y. Cellulose nanoparticles as modifiers for rheology and fluid loss in bentonite water-based fluids. ACS Appl. Mater. Interfaces 2015, 7 (8), 50065016.

(49) Dimic-Misic, K.; Ridgway, C.; Maloney, T.; Paltakari, J.; Gane, $\mathrm{P}$. Influence on pore structure of micro/nanofibrillar cellulose in pigmented coating formulations. Transp. Porous Media 2014, 103 (2), 155-179.

(50) Liu, Y.; Li, B.; Li, Y.; Ocampo, H. Interfacial properties of nano $\mathrm{TiO}_{2}$ and cellulose paper coating. J. Bioresour. Bioprod. 2016, 1 (4), 205-212. 